P085 RELATIVE CONTRIBUTION OF GENERAL PRACTICES AND SEXUAL HEALTH CENTRES IN STI CONSULTATIONS IN THE NETHERLANDS

${ }^{1}$ Isabel Slurink, ${ }^{2}$ Karlijn Groen, ${ }^{2} \mathrm{C}$ Arguedas Flores, ${ }^{3}$ Michelle Kroone, ${ }^{4}$ Arjan Hogewoning, ${ }^{5}$ Alewijn Ott, ${ }^{6}$ Wim Niessen, ${ }^{7}$ Nicole Dukers-Muijrers, ${ }^{8}$ Christian Hoebe, ${ }^{9}$ Hannelore Götz* ${ }^{10}$ Bram Meima, ${ }^{11}$ Femke Koedijk, ${ }^{11}$ Karlijn Kampman, ${ }^{1} J a n$ Van Bergen. ${ }^{1}$ National Institute for Public Health and the Environment (RIVM), Centre For Infectious Disease Control, Bilthoven, Netherlands; ${ }^{2}$ Amsterdam University Medical Center, Department of General Practice, Amsterdam, Netherlands; ${ }^{3}$ Public Health Service Amsterdam, Infectious Diseases, Amsterdam, Netherlands; ${ }^{4}$ Public Health Service Amsterdam, Amsterdam University Medical Center (UMC), National Institute of Public Health and the Environment (RIVM), Infectious Diseases, Infection and Immunity Institute (AI and II), Epidemiology and Surveillance Unit, Amsterdam, Netherlands; ${ }^{5}$ Certe, Medical Microbiology, Groningen, Netherlands; ${ }^{6}$ Municipal Public Health Service Groningen, Groningen, Netherlands; ${ }^{7}$ Public Health Service South Limburg, Sexual Health, Infectious Diseases and Environmental Health, Heerlen, Netherlands; ${ }^{8}$ Public Health Service South Limburg, Maastricht University Medical Center (MUMC), Sexual Health, Infectious Diseases and Environmental Health, Medical Microbiology, Care and Public Health Research Institute (CAPHRI), Heerlen, Netherlands; ${ }^{9} 1$ Public Health Service Rotterdam Rijnmond; 2 Erasmus MC University Medical Center Rotterdam; 3 National Institute for Public Health and the Environment (RIVM), 1 Public Health/Sexual Health; 2 Department of Public Health; 3 Epidemiology and Surveillance Unit, Centre For Infectious Disease Control, Rotterdam, Netherlands; ${ }^{10} 1$ Municipality of Rotterdam; 2 Public Health Service Rotterdam-Rijnmond, 2 Research And Business Intelligence; 2 Public Health, Rotterdam, Netherlands; "11Public Health Service Twente, Sexual Health, Twente, Netherlands

10.1136/sextrans-2019-sti.280

Background Dutch estimates suggest that general practitioners (GPs) perform two-third of all STI consultations, and centres for sexual health (CSH) account for most of the remaining consultations. Yet, a comparison of consultation rates by both providers is hampered due to separate surveillance systems. We assessed the distribution of STI consultations (defined as urogenital Chlamydia trachomatis (CT) test-rates) in five regions.

Methods Data (2011 to 2016) on laboratory testing and diagnosis of urogenital CT were retrieved from CSH at public health services and laboratories in five Dutch geographical regions, aggregated by sex and age category (1524 and 25-64 years). Regions included Amsterdam, Rotterdam, Northeast -Netherlands (N-NL), Twente and Maastricht. Using negative binomial regression, we tested for time-trends in CT test-rate per 10,000 population at GPs and $\mathrm{CSH}$ within five regions and differences between the providers by region.

Results The five regions included in this study varied from mostly rural (N-NL) to highly urban (Amsterdam and Rotterdam). The relative contribution of GPs in consultation rate decreased over time in Amsterdam (60.0-47.8\%), Twente (78.6-61.4\%), Maastricht (59.2-47.2\%), N-NL (82.4-76.9\%), but not in Rotterdam (65.4-67.0\%). This decrease resulted from an increase in all $\mathrm{CSH}$ consultation rates and a slight decrease in GP consultation rate (except for Rotterdam). Women and persons aged 25-64 years were more likely to be tested by GPs compared to CSH (relative risks of 5 regions ranging from $1.47-4.76 ; 1.58-7.43$ respectively). All regions combined, the average yearly positivity rate was $9.2 \%$ at the GP and $10.7 \%$ at the $\mathrm{CSH}$.

Conclusion GPs are still a major contributor in STI consultations, yet the CSH- contribution has increased between 2011 and 2016. In urban regions, the CSH has a considerable role, whereas in rural areas the GP provides most consultations. An explanation for differences may be CSH accessibility.

Disclosure No significant relationships.

\section{P086 SOCIAL BONDS AS A STRATEGY OF PRACTICAL ASSISTANCE FOR SEX WORKER HEALTHCARE}

${ }^{1}$ Ana Amélia Bones* ${ }^{2}$ Carlos Andres Casas, ${ }^{3}$ Mcarthur Barrow, ${ }^{1}$ Silvio Cazella, ${ }^{4}$ Airton Stein. ${ }^{1}$ Health Federal University from Porto Alegre, Health Informatics Post Graduation Program, Porto Alegre, Brazil; ${ }^{2}$ Health Federal University from Porto Alegre, Medicine Academic, Bogota, Colombia; ${ }^{3}$ Municipal Secretary of Health of Porto Alegre, Primary Health Care Trainee Program, St. Philip, Barbados; ${ }^{4}$ Health Federal University from Porto Alegre, Health Science Post Graduation Program, Porto Alegre, Brazil

\subsection{6/sextrans-2019-sti.281}

Background Sex Workers (SW) present greater vulnerability to sexually transmitted infections. There is a need to stimulate an active screening in the vulnerable population in the área covered by a Primary Health Care (PHC) team. The main goal of this study is to present an alternative healthcare assistance approach, applied to a specific area where a high index of SW can be found within the Southern Region of Brazil.

Methods Experience case from a Brazilian PHU regarding adhesion to the unit, local interventions, and rapid test triage in SW was carried out in the health post, in order to promote sexual and reproductive healthcare in an interval of 5 years.

Results Work related to the depicted area started with weekly visits as an approach strategy while offering health care services. Later on, a family planning was conducted and it included provinding access to condom. The SW didn't accept the activities proposed by the PHC. The acceptance of the professional team amongst the community was slow, there was mistrust on the real interest for SW healthcare. The continuity of care was regarded as one os the strategies that was more effective to improve the quality of life of SW. Adhesion to prenatal services, offer of family planning and application of rapid testing for STIs were the most relevant activities that had been implemented. Within 173 applied tests, 135(78\%) were women, of these 27 $(15,6 \%)$ presented pregnancy, and $11(6,3 \%)$ were trans women. $38(21,9 \%)$ were men. Within these tests, $10(6,3 \%)$ were positive for HIV, 35(20,3\%) for syphilis and $1(0,6 \%)$ for HCV.

Conclusion An effective intervention was possible when the health professional had the following scope: action at local level, engagement of local commerce, improve housing, and to involve the whole community in promoting better healthcare. Future studies should be designed to investigate the role of PHC in the SW healthcare.

Disclosure No significant relationships. 